\title{
Erratum: Excitation of Chirping Whistler Waves in a Laboratory Plasma [Phys. Rev. Lett. 114, 245002 (2015)]
}

\author{
B. Van Compernolle, X. An, J. Bortnik, R. M. Thorne, P. Pribyl, and W. Gekelman \\ (Received 25 May 2016; published 26 July 2016)
}

DOI: 10.1103/PhysRevLett.117.059901

We reported on a number of whistler waves that were generated by a gyrating electron beam in a background magnetoplasma. A variety of wave forms (both broadband and discrete frequency rising and falling tones) were observed below the electron cyclotron frequency.

In subsequent experiments, we noticed that intense waves above the cyclotron frequency were also generated and it appeared that some of the original observations of discrete chirping whistlers were contaminated by aliasing of the high harmonics of this mode. In order to verify our results the experiment was carefully repeated at a higher digitization rate and with physical antialiasing filters in place, which mitigated the aliasing issue.

We found that the chirping whistler waves were also present in the second round of experiments. Although similar emission forms were observed they were not exactly the same as previously reported. The main difference is that the discrete whistler emissions found in the second round of experiments are shorter lived, but the magnitudes are the same.

Examples of discrete frequency chirping emissions in the whistler range, replacing Figs. 3(b)-3(e) and Fig. 2(c) of the Letter, are shown in Fig. 1 of this Erratum. The discrete emissions were carefully identified and verified to be whistler waves through a cross-correlation measurement analysis similar to the analysis of Ref. [1]. The parametric dependence of the occurrence rate for discrete frequency chirping whistler waves, as shown in Fig. 4 of the Letter, remains valid.

The rest of the Letter and the conclusions are unaffected.
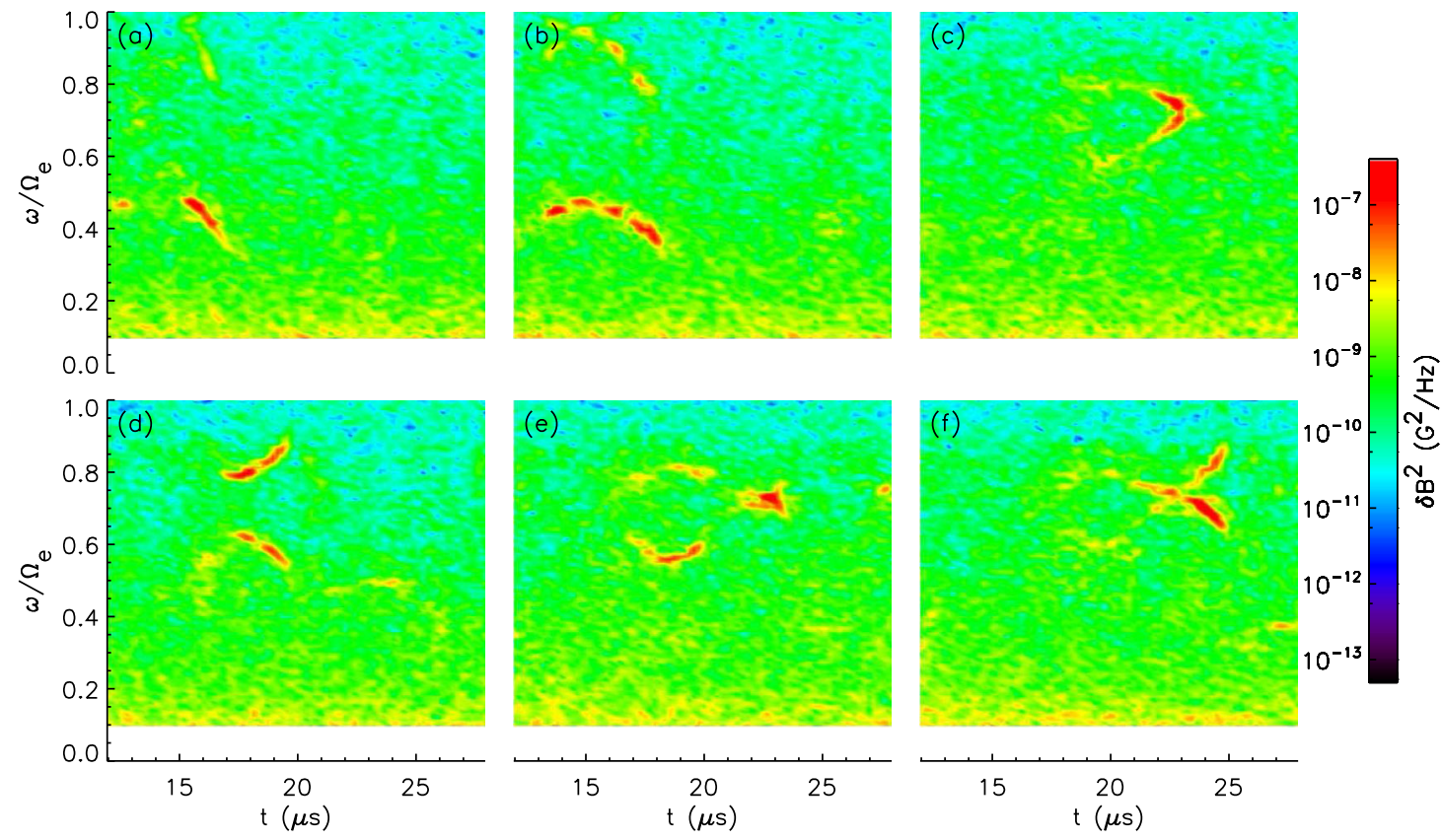

FIG. 1. Spectrograms of types of beam generated wave emissions, ranging from falling tones, hooks, and simultaneous rising and falling tones to crossing falling and rising tones. The fast electron energy is $3 \mathrm{keV}$ for all cases, and $\omega_{p e} / \Omega_{e}=2.6,2.6,2.9,2.6,2.9,2.9$ and $n_{b} / n_{0}(\%)=1.8,1.8,1.5,1.8,1.5,1.5$ for panels (a)-(f), respectively.

[1] X. An, B. Van Compernolle, J. Bortnik, R. M. Thorne, L. Chen, and W. Li, Resonant excitation of whistler waves by a helical electron beam, Geophys. Res. Lett. 43, 2413 (2016). 